\title{
Micellization of Lactosylammonium Surfactants with Different Counter Ions and Their Interaction with DNA
}

\author{
Lifei Zhang, Yanyan Dong, Xiaohong Zhang, Xia Guo* \\ School of Chemistry and Chemical Engineering, Yangzhou University, Yangzhou, \\ Jiangsu, 225002, P. R. China
}

\section{Table Caption}

Table S1 Conductivities ( $\kappa)$ for aqueous solutions of surfactants $\underline{\mathbf{2 a}}$ and $\underline{\mathbf{2 b}}$ as a function of surfactant molality $(\mathrm{m})$ in water at Pressure $\mathrm{p}=0.1 \mathrm{MPa}$

Table S2 Surface tensions $(\gamma)$ for aqueous solutions of surfactants $\underline{\mathbf{2} \mathbf{a}}$ and $\underline{\mathbf{2 b}}$ as a function of surfactant molality $(\mathrm{m})$ in water at Pressure $\mathrm{p}=0.1 \mathrm{MPa}$

Table S3 Surface tension $(\gamma)$ and conductivity ( $\kappa)$ for aqueous solution of surfactant $\underline{\mathbf{3 a}}$ as a function of surfactant molality $(\mathrm{m})$ in water at Pressure $\mathrm{p}=0.1 \mathrm{MPa}$

Table S4 Surface tension $(\gamma)$ and conductivity $(\kappa)$ for aqueous solution of surfactant $\underline{\mathbf{3 b}}$ as a function of surfactant molality $(\mathrm{m})$ in water at Pressure $\mathrm{p}=0.1 \mathrm{MPa}$

Table S5 Dependence of $I_{1} / I_{3}$ of pyrene on molality of surfactant $\underline{\mathbf{2 a}}$ and $\underline{\mathbf{2 b}}$ in water in the absence and presence of DNA

Table S6 Dependence of $I_{1} / I_{3}$ of pyrene on molality of surfactant $\underline{\mathbf{3 a}}$ and $\underline{\mathbf{3 b}}$ in water in the absence and presence of DNA

Table S7 Fluorescence intensities (FL) of EB at $595 \mathrm{~nm}$ in aqueous solution of surfactant ㄹa with and without DNA and fluorescence intensities of surfactant aqueous solutions

Table S8 Fluorescence intensities (FL) of EB at $595 \mathrm{~nm}$ in aqueous solution of surfactant $\underline{\mathbf{2 b}}$ with and without DNA and fluorescence intensities of surfactant aqueous solutions 
Table S9 Fluorescence intensities (FL) of EB at $595 \mathrm{~nm}$ in aqueous solution of surfactant $\underline{\mathbf{3 a}}$ with and without DNA and fluorescence intensities of surfactant aqueous solutions

Table S10 Fluorescence intensities (FL) of EB at $595 \mathrm{~nm}$ in aqueous solution of surfactant $\underline{\mathbf{3 b}}$ with and without DNA and fluorescence intensities of surfactant aqueous solutions

Figure Caption

Figure S1 ${ }^{1} \mathrm{H}$ NMR spectrum of $\underline{\mathbf{2} \mathbf{a}}$ in $\mathrm{D}_{2} \mathrm{O}$

Figure S2 ${ }^{1} \mathrm{H}$ NMR spectrum of $\underline{\mathbf{2 b}}$ in $\mathrm{D}_{2} \mathrm{O}$ 
Table S1 Conductivities ( $\kappa)$ for aqueous solutions of surfactants $\underline{\mathbf{2 a}}$ and $\underline{\mathbf{2 b}}$ as a function of surfactant molality $(\mathrm{m})$ in water at Pressure $p=0.1 \mathrm{MPa}^{\text {a }}$

\begin{tabular}{|c|c|c|c|c|c|c|c|}
\hline surfactant & $\mathrm{m} / \mathrm{mmol} \mathrm{kg}^{-1}$ & $\mathrm{t} /{ }^{\circ} \mathrm{C}$ & $\kappa / \mu \mathrm{s} \cdot \mathrm{cm}^{-1}$ & surfactant & $\mathrm{m} / \mathrm{mmol} / \mathrm{kg}^{-1}$ & $\mathrm{t} /{ }^{\circ} \mathrm{C}$ & $\kappa / \mu \mathrm{s} \cdot \mathrm{cm}^{-1}$ \\
\hline $2 a$ & 0.00 & 25.0 & 2.22 & $2 b$ & 1.59 & 25.0 & 60.4 \\
\hline $2 a$ & 1.59 & 25.0 & 59.7 & $2 b$ & 3.17 & 25.0 & 112.6 \\
\hline $2 a$ & 3.17 & 25.0 & 115.5 & $2 b$ & 3.96 & 25.0 & 137.1 \\
\hline $2 a$ & 4.74 & 25.0 & 168.3 & $2 b$ & 4.74 & 25.0 & 158.9 \\
\hline $2 a$ & 5.52 & 25.0 & 194.3 & $2 b$ & 5.52 & 25.0 & 181.8 \\
\hline $2 a$ & 6.30 & 25.0 & 227 & $2 b$ & 6.30 & 25.0 & 212 \\
\hline $2 a$ & 7.07 & 25.0 & 251 & $2 b$ & 7.07 & 25.0 & 230 \\
\hline $2 a$ & 7.84 & 25.0 & 271 & $2 b$ & 7.84 & 25.0 & 245 \\
\hline $2 a$ & 8.61 & 25.0 & 288 & $2 b$ & 8.61 & 25.0 & 254 \\
\hline $2 a$ & 9.38 & 25.0 & 302 & $2 b$ & 9.38 & 25.0 & 261 \\
\hline $2 a$ & 10.14 & 25.0 & 310 & $2 b$ & 10.14 & 25.0 & 265 \\
\hline $2 a$ & 10.89 & 25.0 & 318 & $2 b$ & 10.89 & 25.0 & 271 \\
\hline $2 a$ & 12.40 & 25.0 & 329 & $2 b$ & 12.40 & 25.0 & 278 \\
\hline $2 a$ & 13.90 & 25.0 & 336 & $2 b$ & 13.90 & 25.0 & 284 \\
\hline $2 a$ & 15.38 & 25.0 & 344 & $2 b$ & 15.38 & 25.0 & 291 \\
\hline $2 a$ & 16.86 & 25.0 & 349 & $2 b$ & 16.86 & 25.0 & 296 \\
\hline $2 a$ & 18.32 & 25.0 & 355 & $2 b$ & 18.32 & 25.0 & 302 \\
\hline $2 a$ & 0.00 & 50.0 & 2.18 & $2 b$ & 19.77 & 25.0 & 309 \\
\hline $2 a$ & 1.59 & 50.0 & 95.8 & $2 b$ & 21.21 & 25.0 & 313 \\
\hline $2 a$ & 3.17 & 50.0 & 163.6 & $2 b$ & 22.64 & 25.0 & 318 \\
\hline $2 a$ & 4.74 & 50.0 & 257 & $2 b$ & 1.59 & 50.0 & 89.8 \\
\hline $2 a$ & 6.30 & 50.0 & 329 & $2 b$ & 3.17 & 50.0 & 165.0 \\
\hline $2 a$ & 7.84 & 50.0 & 387 & $2 b$ & 4.74 & 50.0 & 258 \\
\hline $2 a$ & 9.38 & 50.0 & 428 & $2 b$ & 6.30 & 50.0 & 334 \\
\hline $2 a$ & 10.89 & 50.0 & 457 & $2 b$ & 7.84 & 50.0 & 388 \\
\hline $2 a$ & 12.40 & 50.0 & 479 & $2 b$ & 9.38 & 50.0 & 418 \\
\hline $2 a$ & 13.90 & 50.0 & 494 & $2 b$ & 10.89 & 50.0 & 438 \\
\hline $2 a$ & 15.38 & 50.0 & 509 & $2 b$ & 12.40 & 50.0 & 448 \\
\hline $2 a$ & 16.86 & 50.0 & 519 & $2 b$ & 13.90 & 50.0 & 462 \\
\hline $2 a$ & 18.32 & 50.0 & 528 & $2 b$ & 16.12 & 50.0 & 475 \\
\hline $2 a$ & 20.49 & 50.0 & 543 & $2 b$ & 18.32 & 50.0 & 493 \\
\hline \multirow[t]{2}{*}{$2 a$} & 22.64 & 50.0 & 552 & $2 b$ & 20.49 & 50.0 & 506 \\
\hline & & & & $2 b$ & 22.64 & 50.0 & 521 \\
\hline
\end{tabular}

${ }^{a}$ Standard uncertainties $u$ are $u(\mathrm{t})=0.1^{\circ} \mathrm{C}, u(p)=10 \mathrm{kPa}$, and relative standard uncertainties $u_{\mathrm{r}}$ are $u_{\mathrm{r}}(\mathrm{m})=0.01$ and $u_{\mathrm{r}}(\kappa)=0.05$. 
Table S2 Surface tensions $(\gamma)$ for aqueous solutions of surfactants $\underline{\mathbf{2 a}}$ and $\underline{\mathbf{2 b}}$ as a function of surfactant molality (m) in water at Pressure $p=0.1 \mathrm{MPa}^{\text {a }}$

\begin{tabular}{|c|c|c|c|c|c|c|c|}
\hline surfactant & $\mathrm{m} / \mathrm{mmol} \mathrm{kg}^{-1}$ & $\mathrm{t} /{ }^{\circ} \mathrm{C}$ & $\gamma / \mathrm{mN} \cdot \mathrm{m}^{-1}$ & surfactant & $\mathrm{m} / \mathrm{mmol} \cdot \mathrm{kg}^{-1}$ & $\mathrm{t} /{ }^{\circ} \mathrm{C}$ & $\gamma / \mathrm{mN} \cdot \mathrm{m}^{-1}$ \\
\hline $2 a$ & 0.00 & 25.0 & 71.76 & $2 b$ & 0.00 & 25.0 & 72.71 \\
\hline $2 a$ & 0.50 & 25.0 & 68.12 & $2 b$ & 0.50 & 25.0 & 65.42 \\
\hline $2 a$ & 1.00 & 25.0 & 61.14 & $2 b$ & 1.00 & 25.0 & 57.54 \\
\hline $2 a$ & 1.50 & 25.0 & 55.99 & $2 b$ & 1.50 & 25.0 & 52.44 \\
\hline $2 a$ & 2.00 & 25.0 & 51.91 & $2 b$ & 2.00 & 25.0 & 48.72 \\
\hline $2 a$ & 2.50 & 25.0 & 47.50 & $2 b$ & 2.50 & 25.0 & 46.11 \\
\hline $2 a$ & 3.00 & 25.0 & 44.90 & $2 b$ & 3.00 & 25.0 & 43.65 \\
\hline $2 a$ & 4.00 & 25.0 & 40.37 & $2 b$ & 4.00 & 25.0 & 39.60 \\
\hline $2 a$ & 5.00 & 25.0 & 35.39 & $2 b$ & 5.00 & 25.0 & 36.39 \\
\hline $2 a$ & 6.00 & 25.0 & 32.88 & $2 b$ & 6.00 & 25.0 & 34.24 \\
\hline $2 a$ & 7.00 & 25.0 & 30.63 & $2 b$ & 7.00 & 25.0 & 33.10 \\
\hline $2 a$ & 8.00 & 25.0 & 28.97 & $2 b$ & 9.00 & 25.0 & 32.83 \\
\hline $2 a$ & 9.00 & 25.0 & 28.47 & $2 b$ & 11.00 & 25.0 & 32.93 \\
\hline $2 a$ & 11.00 & 25.0 & 28.33 & $2 b$ & 13.00 & 25.0 & 33.00 \\
\hline $2 a$ & 13.00 & 25.0 & 28.30 & $2 b$ & 15.00 & 25.0 & 33.02 \\
\hline $2 a$ & 16.00 & 25.0 & 28.37 & $2 b$ & 17.00 & 25.0 & 33.05 \\
\hline $2 a$ & 19.00 & 25.0 & 28.35 & $2 b$ & 30.00 & 25.0 & 33.07 \\
\hline $2 a$ & 22.00 & 25.0 & 28.48 & $2 b$ & 0.00 & 50.0 & 68.94 \\
\hline $2 a$ & 0.00 & 50.0 & 68.05 & $2 b$ & 0.40 & 50.0 & 62.97 \\
\hline $2 a$ & 0.40 & 50.0 & 65.73 & $2 b$ & 0.60 & 50.0 & 58.09 \\
\hline $2 a$ & 0.60 & 50.0 & 62.69 & $2 b$ & 1.00 & 50.0 & 52.58 \\
\hline $2 a$ & 0.80 & 50.0 & 59.26 & $2 b$ & 1.40 & 50.0 & 47.88 \\
\hline $2 a$ & 1.00 & 50.0 & 56.85 & $2 b$ & 2.00 & 50.0 & 43.86 \\
\hline $2 a$ & 1.40 & 50.0 & 52.69 & $2 b$ & 2.40 & 50.0 & 41.18 \\
\hline $2 a$ & 2.00 & 50.0 & 47.40 & $2 b$ & 3.00 & 50.0 & 38.37 \\
\hline $2 a$ & 2.40 & 50.0 & 44.70 & $2 b$ & 4.00 & 50.0 & 35.16 \\
\hline $2 a$ & 3.00 & 50.0 & 41.32 & $2 b$ & 5.00 & 50.0 & 32.73 \\
\hline $2 a$ & 4.00 & 50.0 & 37.19 & $2 b$ & 6.00 & 50.0 & 32.17 \\
\hline $2 a$ & 5.00 & 50.0 & 33.30 & $2 b$ & 7.00 & 50.0 & 31.52 \\
\hline $2 a$ & 6.00 & 50.0 & 30.53 & $2 b$ & 8.00 & 50.0 & 31.34 \\
\hline $2 a$ & 7.00 & 50.0 & 28.43 & $2 b$ & 9.00 & 50.0 & 31.53 \\
\hline $2 a$ & 8.00 & 50.0 & 26.79 & $2 b$ & 11.00 & 50.0 & 31.74 \\
\hline $2 a$ & 9.00 & 50.0 & 26.34 & $2 b$ & 13.00 & 50.0 & 31.65 \\
\hline $2 a$ & 11.00 & 50.0 & 25.59 & $2 b$ & 16.00 & 50.0 & 31.86 \\
\hline $2 a$ & 13.00 & 50.0 & 25.63 & $2 b$ & 20.00 & 50.0 & 31.61 \\
\hline $2 a$ & 16.00 & 50.0 & 25.44 & $2 b$ & 30.00 & 50.0 & 31.94 \\
\hline $2 a$ & 20.00 & 50.0 & 25.70 & & & & \\
\hline $2 a$ & 30.00 & 50.0 & 25.69 & & & & \\
\hline
\end{tabular}

${ }^{a}$ Standard uncertainties $u$ are $u(\mathrm{t})=0.1{ }^{\circ} \mathrm{C}, u(p)=10 \mathrm{kPa}, u(\gamma)=0.5 \mathrm{mN} \cdot \mathrm{m}^{-1}$, and relative standard uncertainty $u_{\mathrm{r}}$ is $u_{\mathrm{r}}(\mathrm{m})=0.01$. 
Table S3 Surface tension $(\gamma)$ and conductivity $(\kappa)$ for aqueous solution of surfactant $\underline{\mathbf{3 a}}$ as a function of surfactant molality (m) in water at Pressure $p=0.1 \mathrm{MPa}^{\text {a }}$

\begin{tabular}{cccc|ccc}
\hline surfactant & $\mathrm{m} / \mathrm{mmol}^{\circ} \mathrm{kg}^{-1}$ & $\mathrm{t} /{ }^{\circ} \mathrm{C}$ & $\gamma / \mathrm{mN} \cdot \mathrm{m}^{-1}$ & $\mathrm{~m} / \mathrm{mmol}^{\circ} \mathrm{kg}^{-1}$ & $\mathrm{t} /{ }^{\circ} \mathrm{C}$ & $\kappa / \mu \mathrm{s} \cdot \mathrm{cm}^{-1}$ \\
\hline 3a & 0.00 & 50.0 & 68.57 & 0.00 & 50.0 & 1.534 \\
3a & 0.20 & 50.0 & 67.63 & 0.20 & 50.0 & 10.42 \\
3a & 0.30 & 50.0 & 63.03 & 0.40 & 50.0 & 19.21 \\
3a & 0.40 & 50.0 & 58.77 & 0.60 & 50.0 & 27.2 \\
3a & 0.50 & 50.0 & 53.31 & 0.79 & 50.0 & 35.5 \\
3a & 0.60 & 50.0 & 50.73 & 0.99 & 50.0 & 43.5 \\
3a & 0.70 & 50.0 & 45.91 & 1.19 & 50.0 & 51.2 \\
3a & 0.80 & 50.0 & 42.76 & 1.38 & 50.0 & 58.4 \\
3a & 0.90 & 50.0 & 39.81 & 1.57 & 50.0 & 65.8 \\
3a & 1.00 & 50.0 & 37.73 & 1.77 & 50.0 & 73.1 \\
3a & 1.10 & 50.0 & 35.61 & 1.96 & 50.0 & 80.1 \\
3a & 1.20 & 50.0 & 34.86 & 2.15 & 50.0 & 87.3 \\
3a & 1.60 & 50.0 & 32.47 & 2.34 & 50.0 & 94.2 \\
3a & 2.00 & 50.0 & 31.21 & 2.53 & 50.0 & 101.2 \\
3a & 3.00 & 50.0 & 30.02 & 2.72 & 50.0 & 108.2 \\
3a & 4.00 & 50.0 & 29.74 & 2.91 & 50.0 & 114.8 \\
3a & 6.00 & 50.0 & 29.60 & 3.10 & 50.0 & 120.9 \\
3a & 9.00 & 50.0 & 29.82 & 3.29 & 50.0 & 127.2 \\
3a & 13.00 & 50.0 & 29.82 & & & \\
\hline
\end{tabular}

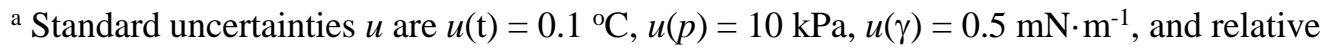
standard uncertainties $u_{\mathrm{r}}$ are $u_{\mathrm{r}}(\mathrm{m})=0.01$ and $u_{\mathrm{r}}(\kappa)=0.05$. 
Table S4 Surface tension $(\gamma)$ and conductivity $(\kappa)$ for aqueous solution of surfactant $\underline{\mathbf{3 b}}$ as a function of surfactant molality (m) in water at Pressure $p=0.1 \mathrm{MPa}^{\text {a }}$

\begin{tabular}{|c|c|c|c|c|c|c|}
\hline surfactant & $\mathrm{m} / \mathrm{mmol} \cdot \mathrm{kg}^{-1}$ & $\mathrm{t} /{ }^{\circ} \mathrm{C}$ & $\gamma / \mathrm{mN} \cdot \mathrm{m}^{-1}$ & $\mathrm{~m} / \mathrm{mmol} \cdot \mathrm{kg}^{-1}$ & $\mathrm{t} /{ }^{\circ} \mathrm{C}$ & $\kappa / \mu \mathrm{s} \cdot \mathrm{cm}^{-1}$ \\
\hline $3 b$ & 0.00 & 50.0 & 68.72 & 0.00 & 50.0 & 2.55 \\
\hline $3 b$ & 0.40 & 50.0 & 58.74 & 0.40 & 50.0 & 18.51 \\
\hline $3 b$ & 0.60 & 50.0 & 52.11 & 0.79 & 50.0 & 33.5 \\
\hline $3 b$ & 0.80 & 50.0 & 48.69 & 1.19 & 50.0 & 48.0 \\
\hline $3 b$ & 1.00 & 50.0 & 45.57 & 1.57 & 50.0 & 60.9 \\
\hline $3 b$ & 1.60 & 50.0 & 37.41 & 1.96 & 50.0 & 73.4 \\
\hline $3 b$ & 2.00 & 50.0 & 34.64 & 2.34 & 50.0 & 86.4 \\
\hline $3 b$ & 2.40 & 50.0 & 32.22 & 2.72 & 50.0 & 98.6 \\
\hline $3 b$ & 3.00 & 50.0 & 30.32 & 3.10 & 50.0 & 108.3 \\
\hline $3 b$ & 4.00 & 50.0 & 27.9 & 3.47 & 50.0 & 117.9 \\
\hline $3 b$ & 5.00 & 50.0 & 27.34 & 3.85 & 50.0 & 127.6 \\
\hline $3 b$ & 7.00 & 50.0 & 26.62 & 4.21 & 50.0 & 136.6 \\
\hline $3 b$ & 11.00 & 50.0 & 26.56 & 4.58 & 50.0 & 144.6 \\
\hline $3 b$ & 16.00 & 50.0 & 26.21 & 4.94 & 50.0 & 152.5 \\
\hline $3 b$ & 20.00 & 50.0 & 26.19 & 5.30 & 50.0 & 159.2 \\
\hline $3 b$ & 30.00 & 50.0 & 26.22 & 5.66 & 50.0 & 165.6 \\
\hline $3 b$ & & & & 6.02 & 50.0 & 172.2 \\
\hline $3 b$ & & & & 6.37 & 50.0 & 178.8 \\
\hline $3 b$ & & & & 6.72 & 50.0 & 183.9 \\
\hline $3 b$ & & & & 7.06 & 50.0 & 189.7 \\
\hline
\end{tabular}

${ }^{\text {a }}$ Standard uncertainties $u$ are $u(\mathrm{t})=0.1{ }^{\circ} \mathrm{C}, u(p)=10 \mathrm{kPa}, u(\gamma)=0.5 \mathrm{mN} \cdot \mathrm{m}^{-1}$, and relative standard uncertainties $u_{\mathrm{r}}$ are $u_{\mathrm{r}}(\mathrm{m})=0.01$ and $u_{\mathrm{r}}(\kappa)=0.05$. 
Table S5 Dependence of $I_{1} / I_{3}$ of pyrene on molality of surfactant $\underline{\mathbf{2} \mathbf{a}}$ and $\underline{\mathbf{2 b}}$ in water in the absence and presence of DNA ${ }^{\mathrm{a}}$

\begin{tabular}{|c|c|c|c|c|c|c|c|}
\hline surfactant & $\mathrm{m}_{\mathrm{A}}^{\mathrm{b}} / \mathrm{mmol} \cdot \mathrm{kg}^{-1}$ & $\mathrm{~m}_{\mathrm{B}}^{\mathrm{c}} / \mathrm{mmol} \cdot \mathrm{kg}^{-1}$ & $\mathrm{I}_{1} / \mathrm{I}_{3}$ & surfactant & $\mathrm{m}_{\mathrm{A}}^{\mathrm{b}} / \mathrm{mmol} \cdot \mathrm{kg}^{-1}$ & $\mathrm{~m}_{\mathrm{B}}^{\mathrm{c}} / \mathrm{mmol} \cdot \mathrm{kg}^{-1}$ & $\mathrm{I}_{1} / \mathrm{I}_{3}$ \\
\hline $2 a$ & 0.010 & 0.00 & 1.73 & $2 b$ & 0.010 & 0.00 & 1.78 \\
\hline $2 a$ & 0.025 & 0.00 & 1.73 & $2 b$ & 0.025 & 0.00 & 1.77 \\
\hline $2 a$ & 0.050 & 0.00 & 1.73 & $2 b$ & 0.050 & 0.00 & 1.78 \\
\hline $2 a$ & 0.10 & 0.00 & 1.73 & $2 b$ & 0.10 & 0.00 & 1.77 \\
\hline $2 a$ & 0.25 & 0.00 & 1.73 & $2 b$ & 0.25 & 0.00 & 1.76 \\
\hline $2 a$ & 1.00 & 0.00 & 1.72 & $2 b$ & 1.00 & 0.00 & 1.75 \\
\hline $2 a$ & 2.00 & 0.00 & 1.69 & $2 b$ & 2.00 & 0.00 & 1.74 \\
\hline $2 a$ & 3.00 & 0.00 & 1.67 & $2 b$ & 3.00 & 0.00 & 1.7 \\
\hline $2 a$ & 4.50 & 0.00 & 1.59 & $2 b$ & 3.50 & 0.00 & 1.62 \\
\hline $2 a$ & 5.00 & 0.00 & 1.53 & $2 b$ & 4.00 & 0.00 & 1.50 \\
\hline $2 a$ & 5.50 & 0.00 & 1.32 & $2 b$ & 4.50 & 0.00 & 1.29 \\
\hline $2 a$ & 6.00 & 0.00 & 1.21 & $2 b$ & 5.00 & 0.00 & 1.19 \\
\hline $2 a$ & 7.00 & 0.00 & 1.09 & $2 b$ & 7.00 & 0.00 & 1.1 \\
\hline $2 a$ & 10.00 & 0.00 & 1.06 & $2 b$ & 10.00 & 0.00 & 1.08 \\
\hline $2 a$ & 14.00 & 0.00 & 1.05 & $2 b$ & 14.00 & 0.00 & 1.06 \\
\hline $2 a$ & 20.00 & 0.00 & 1.05 & $2 b$ & 20.00 & 0.00 & 1.06 \\
\hline $2 a$ & 30.00 & 0.00 & 1.04 & $2 b$ & 30.00 & 0.00 & 1.06 \\
\hline $2 a$ & 0.00 & 0.12 & 1.76 & $2 b$ & 0.00 & 0.12 & 1.76 \\
\hline $2 a$ & 0.010 & 0.12 & 1.76 & $2 b$ & 0.010 & 0.12 & 1.76 \\
\hline $2 a$ & 0.020 & 0.12 & 1.75 & $2 b$ & 0.020 & 0.12 & 1.73 \\
\hline $2 a$ & 0.030 & 0.12 & 1.75 & $2 b$ & 0.040 & 0.12 & 1.74 \\
\hline $2 a$ & 0.050 & 0.12 & 1.74 & $2 b$ & 0.10 & 0.12 & 1.69 \\
\hline $2 a$ & 0.10 & 0.12 & 1.73 & $2 b$ & 0.20 & 0.12 & 1.62 \\
\hline $2 a$ & 0.20 & 0.12 & 1.67 & $2 b$ & 0.30 & 0.12 & 1.55 \\
\hline $2 a$ & 0.30 & 0.12 & 1.58 & $2 b$ & 0.50 & 0.12 & 1.53 \\
\hline $2 a$ & 0.50 & 0.12 & 1.53 & $2 b$ & 1.00 & 0.12 & 1.51 \\
\hline $2 a$ & 1.00 & 0.12 & 1.48 & $2 b$ & 2.00 & 0.12 & 1.51 \\
\hline $2 a$ & 2.00 & 0.12 & 1.40 & $2 b$ & 2.50 & 0.12 & 1.50 \\
\hline $2 a$ & 3.00 & 0.12 & 1.34 & $2 b$ & 3.00 & 0.12 & 1.40 \\
\hline $2 a$ & 4.00 & 0.12 & 1.26 & $2 b$ & 4.00 & 0.12 & 1.23 \\
\hline $2 a$ & 5.00 & 0.12 & 1.19 & $2 b$ & 5.00 & 0.12 & 1.15 \\
\hline $2 a$ & 6.00 & 0.12 & 1.14 & $2 b$ & 6.00 & 0.12 & 1.11 \\
\hline $2 a$ & 8.00 & 0.12 & 1.08 & $2 b$ & 8.00 & 0.12 & 1.08 \\
\hline $2 a$ & 10.00 & 0.12 & 1.06 & $2 b$ & 10.00 & 0.12 & 1.07 \\
\hline $2 a$ & 12.00 & 0.12 & 1.05 & $2 b$ & 20.00 & 0.12 & 1.06 \\
\hline $2 a$ & 16.00 & 0.12 & 1.05 & $2 b$ & 30.00 & 0.12 & 1.05 \\
\hline $2 a$ & 20.00 & 0.12 & 1.04 & & & & \\
\hline $2 a$ & 25.00 & 0.12 & 1.05 & & & & \\
\hline
\end{tabular}

${ }^{\mathrm{a}}$ Relative standard uncertainties $u_{\mathrm{r}}$ are $u_{\mathrm{r}}(\mathrm{m})=0.01$ and $u_{\mathrm{r}}\left(\mathrm{I}_{1} / \mathrm{I}_{3}\right)=0.01 .{ }^{\mathrm{b}} \mathrm{m}_{\mathrm{A}}$ is the molality of surfactant in water. ${ }^{c} m_{B}$ is the molality of DNA in water. 
Table S6 Dependence of $\mathrm{I}_{1} / \mathrm{I}_{3}$ of pyrene on molality of surfactant $\underline{\mathbf{3 a}}$ and $\underline{\mathbf{3 b}}$ in water in the absence and presence of DNA ${ }^{\text {a }}$

\begin{tabular}{|c|c|c|c|c|c|c|c|}
\hline surfactant & $\mathrm{m}_{\mathrm{A}}^{\mathrm{b}} / \mathrm{mmol} \cdot \mathrm{kg}^{-1}$ & $\mathrm{~m}_{\mathrm{B}}^{\mathrm{c}} / \mathrm{mmol} \cdot \mathrm{kg}^{-1}$ & $\mathrm{I}_{1} / \mathrm{I}_{3}$ & surfactant & $\mathrm{m}_{\mathrm{A}}^{\mathrm{b}} / \mathrm{mmol} \cdot \mathrm{kg}^{-1}$ & $\mathrm{~m}_{\mathrm{B}}^{\mathrm{c}} / \mathrm{mmol} \cdot \mathrm{kg}^{-1}$ & $\mathrm{I}_{1} / \mathrm{I}_{3}$ \\
\hline $3 a$ & 0.010 & 0.00 & 1.68 & $3 b$ & 0.010 & 0.00 & 1.69 \\
\hline $3 a$ & 0.020 & 0.00 & 1.68 & $3 b$ & 0.020 & 0.00 & 1.68 \\
\hline $3 a$ & 0.030 & 0.00 & 1.68 & $3 b$ & 0.030 & 0.00 & 1.68 \\
\hline $3 a$ & 0.050 & 0.00 & 1.68 & $3 b$ & 0.050 & 0.00 & 1.73 \\
\hline $3 a$ & 0.10 & 0.00 & 1.69 & $3 b$ & 0.10 & 0.00 & 1.68 \\
\hline $3 a$ & 0.20 & 0.00 & 1.69 & $3 b$ & 0.20 & 0.00 & 1.69 \\
\hline $3 a$ & 0.30 & 0.00 & 1.70 & $3 b$ & 0.30 & 0.00 & 1.72 \\
\hline $3 a$ & 0.50 & 0.00 & 1.69 & $3 b$ & 0.50 & 0.00 & 1.70 \\
\hline $3 a$ & 0.80 & 0.00 & 1.66 & $3 b$ & 0.80 & 0.00 & 1.68 \\
\hline $3 a$ & 1.00 & 0.00 & 1.57 & $3 b$ & 1.00 & 0.00 & 1.62 \\
\hline $3 a$ & 1.20 & 0.00 & 1.39 & $3 b$ & 1.40 & 0.00 & 1.55 \\
\hline $3 a$ & 1.50 & 0.00 & 1.29 & $3 b$ & 1.60 & 0.00 & 1.41 \\
\hline $3 a$ & 2.00 & 0.00 & 1.23 & $3 b$ & 2.00 & 0.00 & 1.28 \\
\hline $3 a$ & 3.00 & 0.00 & 1.21 & $3 b$ & 3.00 & 0.00 & 1.14 \\
\hline $3 a$ & 5.00 & 0.00 & 1.1 & $3 b$ & 5.00 & 0.00 & 1.03 \\
\hline $3 a$ & 10.00 & 0.00 & 1.07 & $3 b$ & 7.00 & 0.00 & 1.03 \\
\hline $3 a$ & 15.00 & 0.00 & 1.03 & $3 b$ & 9.00 & 0.00 & 1.01 \\
\hline $3 a$ & 0.000 & 0.00 & 1.69 & $3 b$ & 0.00 & 0.12 & 1.71 \\
\hline $3 a$ & 0.010 & 0.00 & 1.67 & $3 b$ & 0.010 & 0.12 & 1.71 \\
\hline $3 a$ & 0.020 & 0.12 & 1.70 & $3 b$ & 0.020 & 0.12 & 1.70 \\
\hline $3 a$ & 0.030 & 0.12 & 1.70 & $3 b$ & 0.030 & 0.12 & 1.70 \\
\hline $3 a$ & 0.050 & 0.12 & 1.69 & $3 b$ & 0.050 & 0.12 & 1.70 \\
\hline $3 a$ & 0.10 & 0.12 & 1.69 & $3 b$ & 0.10 & 0.12 & 1.67 \\
\hline $3 a$ & 0.20 & 0.12 & 1.69 & $3 b$ & 0.20 & 0.12 & 1.61 \\
\hline $3 a$ & 0.30 & 0.12 & 1.64 & $3 b$ & 0.30 & 0.12 & 1.58 \\
\hline $3 a$ & 0.50 & 0.12 & 1.58 & $3 b$ & 0.50 & 0.12 & 1.52 \\
\hline $3 a$ & 1.00 & 0.12 & 1.53 & $3 b$ & 0.70 & 0.12 & 1.54 \\
\hline $3 a$ & 1.20 & 0.12 & 1.47 & $3 b$ & 1.00 & 0.12 & 1.56 \\
\hline $3 a$ & 1.50 & 0.12 & 1.36 & $3 b$ & 1.30 & 0.12 & 1.54 \\
\hline $3 a$ & 2.00 & 0.12 & 1.23 & $3 b$ & 1.50 & 0.12 & 1.47 \\
\hline $3 a$ & 3.00 & 0.12 & 1.17 & $3 b$ & 1.60 & 0.12 & 1.39 \\
\hline $3 a$ & 3.50 & 0.12 & 1.14 & $3 b$ & 1.80 & 0.12 & 1.29 \\
\hline $3 a$ & 5.00 & 0.12 & 1.09 & $3 b$ & 2.00 & 0.12 & 1.20 \\
\hline $3 a$ & 6.00 & 0.12 & 1.06 & $3 b$ & 3.00 & 0.12 & 1.10 \\
\hline $3 a$ & 8.00 & 0.12 & 1.06 & $3 b$ & 5.00 & 0.12 & 1.04 \\
\hline $3 a$ & 10.00 & 0.12 & 1.04 & $3 b$ & 7.00 & 0.12 & 1.00 \\
\hline $3 a$ & 14.00 & 0.12 & 1.03 & $3 b$ & 9.00 & 0.12 & 1.00 \\
\hline
\end{tabular}

${ }^{\text {a }}$ Relative standard uncertainties $u_{\mathrm{r}}$ are $u_{\mathrm{r}}(\mathrm{m})=0.01$ and $u_{\mathrm{r}}\left(\mathrm{I}_{1} / \mathrm{I}_{3}\right)=0.01 .{ }^{\mathrm{b}} \mathrm{m}_{\mathrm{A}}$ is the molality of surfactant in water. ${ }^{c} \mathrm{~m}_{\mathrm{B}}$ is the molality of DNA in water. 
Table S7 Fluorescence intensities (FL) of EB at $595 \mathrm{~nm}$ in aqueous solution of surfactant 2a with and without DNA and fluorescence intensities of surfactant aqueous solutions ${ }^{\text {a }}$

\begin{tabular}{|c|c|c|c|c|}
\hline surfactant & $\mathrm{m}_{\mathrm{A}}^{\mathrm{b}} / \mathrm{mmol} \cdot \mathrm{kg}^{-1}$ & $\mathrm{~m}_{\mathrm{B}}^{\mathrm{c}} / \mathrm{mmol} \cdot \mathrm{kg}^{-1}$ & 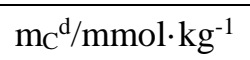 & FL \\
\hline $2 a$ & 0.00 & 0.00 & 0.00 & 3.38 \\
\hline $2 a$ & 0.10 & 0.00 & 0.00 & 3.00 \\
\hline $2 a$ & 1.00 & 0.00 & 0.00 & 3.33 \\
\hline $2 a$ & 3.00 & 0.00 & 0.00 & 3.88 \\
\hline $2 a$ & 5.00 & 0.00 & 0.00 & 5.05 \\
\hline $2 a$ & 8.00 & 0.00 & 0.00 & 6.51 \\
\hline $2 a$ & 12.00 & 0.00 & 0.00 & 6.92 \\
\hline $2 a$ & 16.00 & 0.00 & 0.00 & 8.81 \\
\hline $2 a$ & 0.00 & 0.00 & 0.010 & 40.96 \\
\hline $2 a$ & 0.10 & 0.00 & 0.010 & 63.90 \\
\hline $2 a$ & 1.00 & 0.00 & 0.010 & 82.75 \\
\hline $2 a$ & 3.00 & 0.00 & 0.010 & 71.67 \\
\hline $2 a$ & 5.00 & 0.00 & 0.010 & 91.42 \\
\hline $2 a$ & 8.00 & 0.00 & 0.010 & 84.58 \\
\hline $2 a$ & 12.00 & 0.00 & 0.010 & 67.88 \\
\hline $2 a$ & 16.00 & 0.00 & 0.010 & 76.76 \\
\hline $2 a$ & 0.10 & 0.12 & 0.010 & 600.00 \\
\hline $2 a$ & 0.70 & 0.12 & 0.010 & 575.82 \\
\hline $2 a$ & 0.80 & 0.12 & 0.010 & 443.97 \\
\hline $2 a$ & 1.00 & 0.12 & 0.010 & 202.25 \\
\hline $2 a$ & 1.40 & 0.12 & 0.010 & 99.70 \\
\hline $2 a$ & 2.00 & 0.12 & 0.010 & 48.14 \\
\hline $2 a$ & 3.00 & 0.12 & 0.010 & 75.47 \\
\hline $2 a$ & 5.00 & 0.12 & 0.010 & 113.38 \\
\hline $2 a$ & 6.00 & 0.12 & 0.010 & 157.33 \\
\hline $2 a$ & 7.00 & 0.12 & 0.010 & 205.64 \\
\hline $2 a$ & 8.00 & 0.12 & 0.010 & 222.04 \\
\hline $2 a$ & 12.00 & 0.12 & 0.010 & 216.16 \\
\hline $2 a$ & 16.00 & 0.12 & 0.010 & 209.70 \\
\hline
\end{tabular}

${ }^{\mathrm{a}}$ Relative standard uncertainties $u_{\mathrm{r}}$ are $u_{\mathrm{r}}(\mathrm{m})=0.01$ and $u_{\mathrm{r}}(\mathrm{FL})=0.01 .^{\mathrm{b}} \mathrm{m}_{\mathrm{A}}$ is the molality of surfactant in water. ${ }^{b} \mathrm{~m}_{\mathrm{B}}$ is the molality of DNA in water. ${ }^{c} \mathrm{~m}_{\mathrm{C}}$ is the molality of EB in water. 
Table S8 Fluorescence intensities (FL) of EB at $595 \mathrm{~nm}$ in aqueous solution of surfactant $\underline{\mathbf{2 b}}$ with and without DNA and fluorescence intensities of surfactant aqueous solutions ${ }^{\text {a }}$

\begin{tabular}{|c|c|c|c|c|}
\hline surfactant & $\mathrm{m}_{\mathrm{A}}^{\mathrm{b}} / \mathrm{mmol} \cdot \mathrm{kg}^{-1}$ & $\mathrm{~m}_{\mathrm{B}}^{\mathrm{c}} / \mathrm{mmol} \cdot \mathrm{kg}^{-1}$ & $\mathrm{~m}_{\mathrm{C}} \mathrm{d} / \mathrm{mmol} \cdot \mathrm{kg}^{-1}$ & FL \\
\hline $2 b$ & 0.00 & 0.00 & 0.00 & 3.72 \\
\hline $2 b$ & 0.10 & 0.00 & 0.00 & 4.41 \\
\hline $2 b$ & 1.00 & 0.00 & 0.00 & 4.96 \\
\hline $2 b$ & 2.00 & 0.00 & 0.00 & 4.78 \\
\hline $2 b$ & 3.00 & 0.00 & 0.00 & 5.83 \\
\hline $2 b$ & 5.00 & 0.00 & 0.00 & 8.68 \\
\hline $2 b$ & 8.00 & 0.00 & 0.00 & 12.19 \\
\hline $2 b$ & 12.00 & 0.00 & 0.00 & 16.02 \\
\hline $2 b$ & 0.00 & 0.00 & 0.00 & 39.95 \\
\hline $2 b$ & 0.10 & 0.00 & 0.010 & 44.52 \\
\hline $2 b$ & 1.00 & 0.00 & 0.010 & 56.72 \\
\hline $2 b$ & 2.00 & 0.00 & 0.010 & 55.58 \\
\hline $2 b$ & 3.00 & 0.00 & 0.010 & 57.24 \\
\hline $2 b$ & 5.00 & 0.00 & 0.010 & 53.16 \\
\hline $2 b$ & 8.00 & 0.00 & 0.010 & 53.03 \\
\hline $2 b$ & 12.00 & 0.00 & 0.010 & 73.37 \\
\hline $2 b$ & 0.00 & 0.12 & 0.010 & 600.00 \\
\hline $2 b$ & 0.10 & 0.12 & 0.010 & 519.97 \\
\hline $2 b$ & 0.30 & 0.12 & 0.010 & 304.67 \\
\hline $2 b$ & 0.40 & 0.12 & 0.010 & 219.35 \\
\hline $2 b$ & 0.53 & 0.12 & 0.010 & 103.98 \\
\hline $2 b$ & 1.00 & 0.12 & 0.010 & 84.58 \\
\hline $2 b$ & 2.00 & 0.12 & 0.010 & 71.97 \\
\hline $2 b$ & 3.00 & 0.12 & 0.010 & 93.43 \\
\hline $2 b$ & 4.00 & 0.12 & 0.010 & 208.43 \\
\hline $2 b$ & 5.00 & 0.12 & 0.010 & 222.99 \\
\hline $2 b$ & 8.00 & 0.12 & 0.010 & 259.19 \\
\hline $2 b$ & 12.00 & 0.12 & 0.010 & 257.61 \\
\hline
\end{tabular}

${ }^{\text {a }}$ Relative standard uncertainties $u_{\mathrm{r}}$ are $u_{\mathrm{r}}(\mathrm{m})=0.01$ and $u_{\mathrm{r}}(\mathrm{FL})=0.01 .{ }^{\mathrm{b}} \mathrm{m}_{\mathrm{A}}$ is the molality of surfactant in water. ${ }^{c} \mathrm{~m}_{\mathrm{B}}$ is the molality of DNA in water. ${ }^{\mathrm{d}} \mathrm{m}_{\mathrm{C}}$ is the molality of $\mathrm{EB}$ in water. 
Table S9 Fluorescence intensities (FL) of EB at $595 \mathrm{~nm}$ in aqueous solution of surfactant $\underline{3 \mathbf{a}}$ with and without DNA and fluorescence intensities of surfactant aqueous solutions ${ }^{\text {a }}$

\begin{tabular}{|c|c|c|c|c|}
\hline surfactant & $\mathrm{m}_{\mathrm{A}}^{\mathrm{b}} / \mathrm{mmol} \cdot \mathrm{kg}^{-1}$ & $\mathrm{~m}_{\mathrm{B}}^{\mathrm{c}} / \mathrm{mmol} \cdot \mathrm{kg}^{-1}$ & $\mathrm{~m}_{\mathrm{C}} \mathrm{d} / \mathrm{mmol} \cdot \mathrm{kg}^{-1}$ & FL \\
\hline $3 a$ & 0.00 & 0.00 & 0.00 & 3.12 \\
\hline $3 a$ & 0.10 & 0.00 & 0.00 & 3.27 \\
\hline 3a & 0.50 & 0.00 & 0.00 & 3.65 \\
\hline $3 a$ & 1.00 & 0.00 & 0.00 & 4.41 \\
\hline $3 a$ & 1.50 & 0.00 & 0.00 & 6.37 \\
\hline $3 a$ & 2.00 & 0.00 & 0.00 & 6.85 \\
\hline $3 a$ & 2.50 & 0.00 & 0.00 & 7.83 \\
\hline 3a & 3.00 & 0.00 & 0.00 & 8.25 \\
\hline $3 a$ & 4.00 & 0.00 & 0.00 & 8.05 \\
\hline $3 a$ & 0.00 & 0.00 & 0.010 & 29.46 \\
\hline $3 a$ & 0.10 & 0.00 & 0.010 & 44.48 \\
\hline $3 a$ & 0.50 & 0.00 & 0.010 & 38.80 \\
\hline $3 a$ & 1.00 & 0.00 & 0.010 & 38.79 \\
\hline $3 a$ & 1.50 & 0.00 & 0.010 & 38.97 \\
\hline $3 a$ & 2.00 & 0.00 & 0.010 & 39.39 \\
\hline $3 a$ & 2.50 & 0.00 & 0.010 & 36.93 \\
\hline $3 a$ & 3.00 & 0.00 & 0.010 & 23.93 \\
\hline $3 a$ & 4.00 & 0.00 & 0.010 & 34.35 \\
\hline $3 a$ & 0.00 & 0.12 & 0.010 & 600.00 \\
\hline $3 a$ & 0.050 & 0.12 & 0.010 & 520.43 \\
\hline $3 a$ & 0.10 & 0.12 & 0.010 & 450.59 \\
\hline $3 a$ & 0.25 & 0.12 & 0.010 & 431.45 \\
\hline $3 a$ & 0.30 & 0.12 & 0.010 & 346.57 \\
\hline $3 a$ & 0.50 & 0.12 & 0.010 & 246.12 \\
\hline $3 a$ & 0.70 & 0.12 & 0.010 & 203.73 \\
\hline $3 a$ & 1.00 & 0.12 & 0.010 & 150.20 \\
\hline $3 a$ & 1.30 & 0.12 & 0.010 & 95.15 \\
\hline $3 a$ & 1.50 & 0.12 & 0.010 & 52.23 \\
\hline $3 a$ & 1.80 & 0.12 & 0.010 & 156.67 \\
\hline $3 a$ & 2.00 & 0.12 & 0.010 & 236.81 \\
\hline $3 a$ & 2.50 & 0.12 & 0.010 & 271.21 \\
\hline $3 a$ & 3.00 & 0.12 & 0.010 & 267.95 \\
\hline $3 a$ & 4.00 & 0.12 & 0.010 & 252.85 \\
\hline
\end{tabular}

${ }^{\text {a }}$ Relative standard uncertainties $u_{\mathrm{r}}$ are $u_{\mathrm{r}}(\mathrm{m})=0.01$ and $u_{\mathrm{r}}(\mathrm{FL})=0.01 .{ }^{\mathrm{b}} \mathrm{m}_{\mathrm{A}}$ is the molality of surfactant in water. ${ }^{c} \mathrm{~m}_{B}$ is the molality of DNA in water. ${ }^{d} \mathrm{~m}_{C}$ is the molality of EB in water. 
Table S10 Fluorescence intensities (FL) of EB at $595 \mathrm{~nm}$ in aqueous solution of surfactant $\underline{\mathbf{3 b}}$ with and without DNA and fluorescence intensities of surfactant aqueous solutions ${ }^{a}$

\begin{tabular}{|c|c|c|c|c|}
\hline surfactant & $\mathrm{m}_{\mathrm{A}}^{\mathrm{b}} / \mathrm{mmol} \cdot \mathrm{kg}^{-1}$ & $\mathrm{~m}_{\mathrm{B}}{ }^{\mathrm{c}} / \mathrm{mmol} \cdot \mathrm{kg}^{-1}$ & $\mathrm{~m}_{\mathrm{C}} \mathrm{d} / \mathrm{mmol} \cdot \mathrm{kg}^{-1}$ & FL \\
\hline $3 b$ & 0.00 & 0.00 & 0.00 & 2.97 \\
\hline $3 b$ & 0.10 & 0.00 & 0.00 & 3.53 \\
\hline $3 b$ & 1.00 & 0.00 & 0.00 & 3.97 \\
\hline $3 b$ & 2.00 & 0.00 & 0.00 & 10.45 \\
\hline $3 b$ & 3.00 & 0.00 & 0.00 & 9.80 \\
\hline $3 b$ & 4.00 & 0.00 & 0.00 & 8.00 \\
\hline $3 b$ & 5.00 & 0.00 & 0.00 & 7.82 \\
\hline $3 b$ & 6.00 & 0.00 & 0.00 & 8.66 \\
\hline $3 b$ & 0.00 & 0.00 & 0.010 & 41.28 \\
\hline $3 b$ & 0.10 & 0.00 & 0.010 & 46.36 \\
\hline $3 b$ & 1.00 & 0.00 & 0.010 & 46.27 \\
\hline $3 b$ & 2.00 & 0.00 & 0.010 & 49.71 \\
\hline $3 b$ & 3.00 & 0.00 & 0.010 & 52.45 \\
\hline $3 b$ & 4.00 & 0.00 & 0.010 & 65.60 \\
\hline $3 b$ & 5.00 & 0.00 & 0.010 & 58.75 \\
\hline $3 b$ & 6.00 & 0.00 & 0.010 & 58.21 \\
\hline $3 b$ & 0.00 & 0.12 & 0.010 & 600.00 \\
\hline $3 b$ & 0.20 & 0.12 & 0.010 & 540.01 \\
\hline $3 b$ & 0.30 & 0.12 & 0.010 & 423.05 \\
\hline $3 b$ & 0.40 & 0.12 & 0.010 & 381.95 \\
\hline $3 b$ & 0.70 & 0.12 & 0.010 & 277.21 \\
\hline $3 b$ & 1.00 & 0.12 & 0.010 & 179.77 \\
\hline $3 b$ & 1.30 & 0.12 & 0.010 & 115.69 \\
\hline $3 b$ & 1.70 & 0.12 & 0.010 & 62.14 \\
\hline $3 b$ & 2.00 & 0.12 & 0.010 & 74.65 \\
\hline $3 b$ & 2.30 & 0.12 & 0.010 & 179.51 \\
\hline $3 b$ & 2.50 & 0.12 & 0.010 & 292.00 \\
\hline $3 b$ & 3.00 & 0.12 & 0.010 & 338.35 \\
\hline $3 b$ & 4.00 & 0.12 & 0.010 & 314.85 \\
\hline $3 b$ & 5.00 & 0.12 & 0.010 & 283.87 \\
\hline $3 b$ & 6.00 & 0.12 & 0.010 & 262.04 \\
\hline
\end{tabular}

${ }^{a}$ Relative standard uncertainties $u_{\mathrm{r}}$ are $u_{\mathrm{r}}(\mathrm{m})=0.01$ and $u_{\mathrm{r}}(\mathrm{FL})=0.01 .{ }^{\mathrm{b}} \mathrm{m}_{\mathrm{A}}$ is the molality of surfactant in water. ${ }^{c} m_{B}$ is the molality of DNA in water. ${ }^{d} m_{C}$ is the molality of EB in water. 


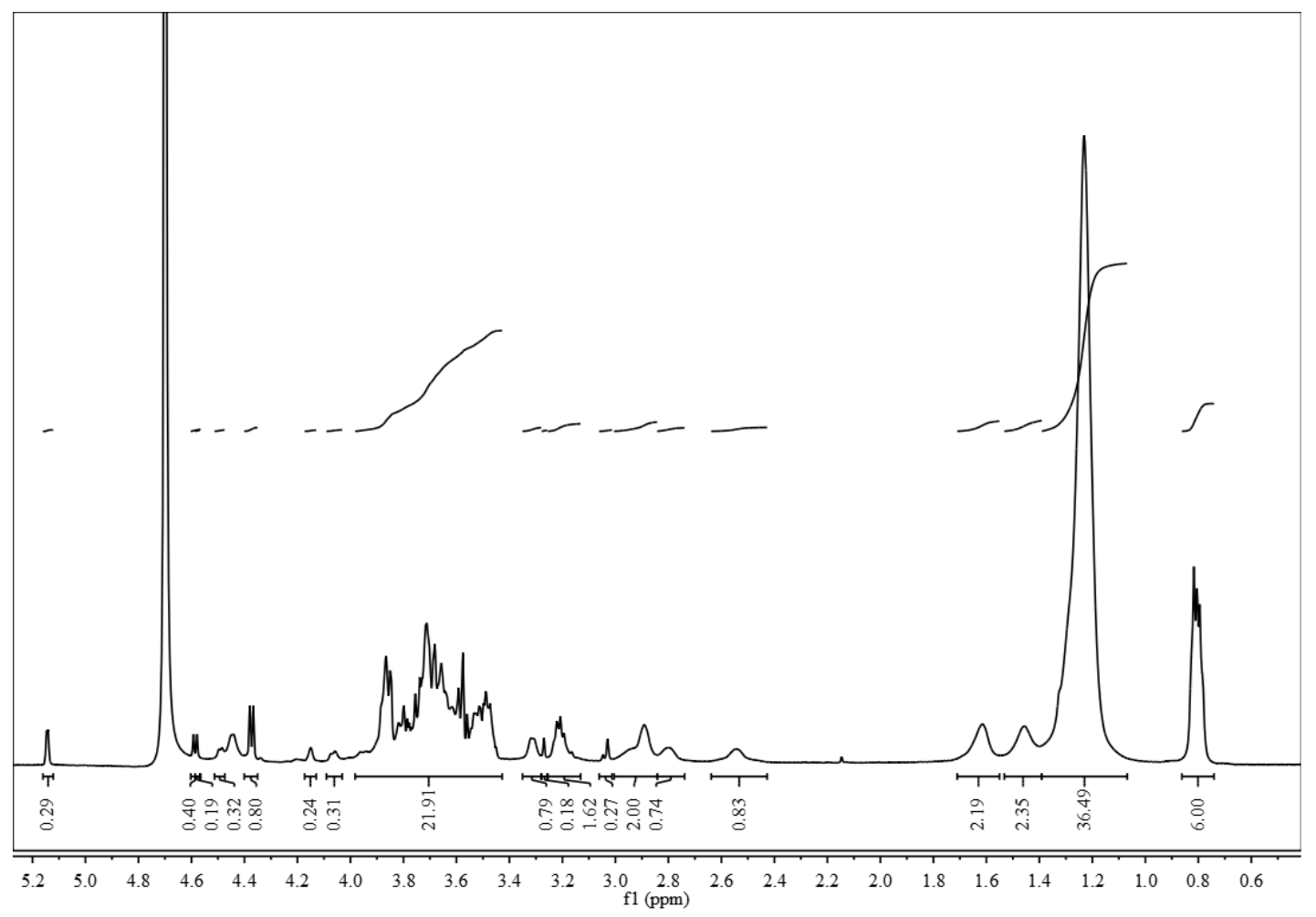

Figure $\mathbf{S 1}{ }^{1} \mathrm{H}$ NMR spectrum of $\underline{\mathbf{2 a}}$ in $\mathrm{D}_{2} \mathrm{O}$ 


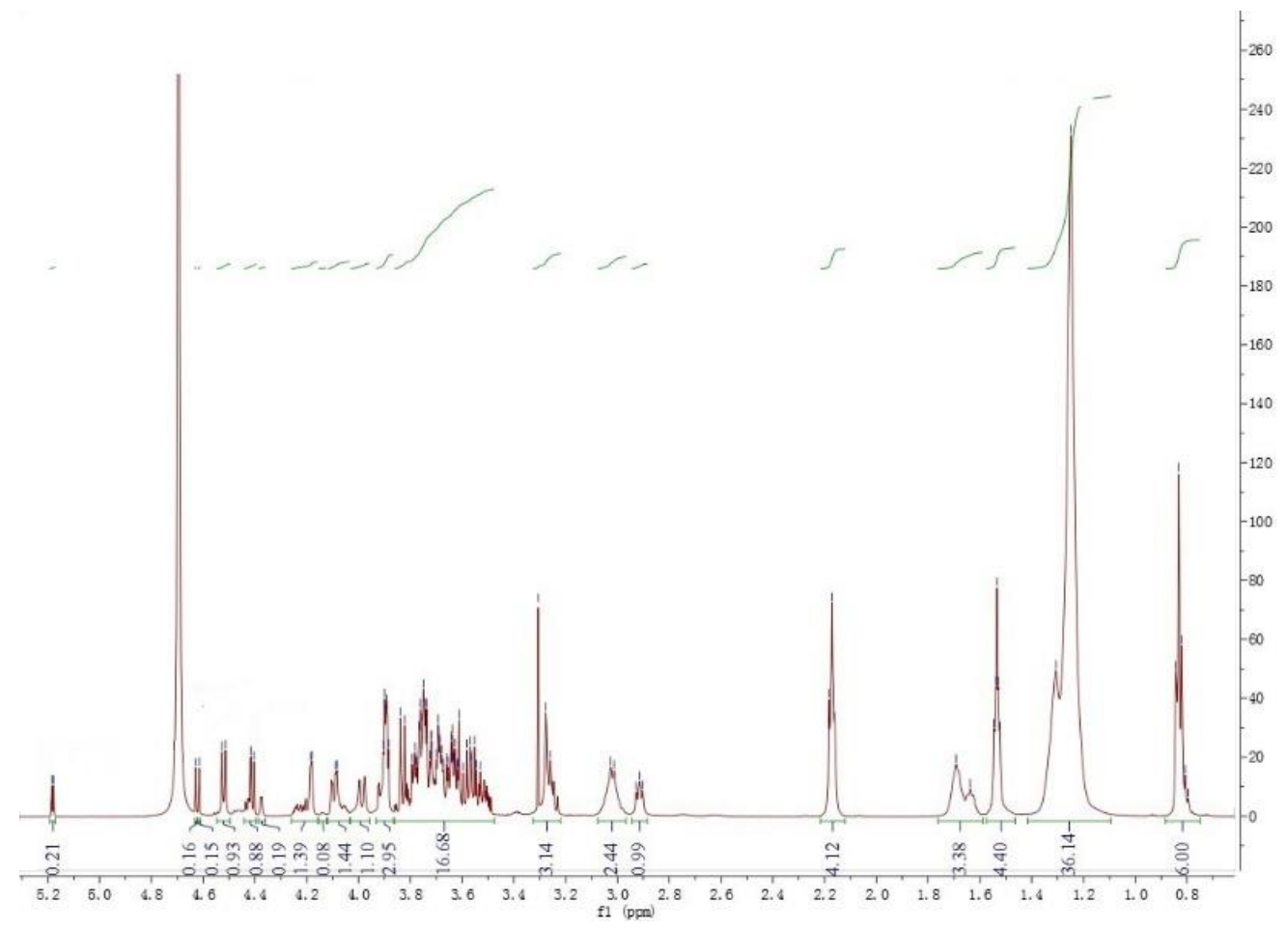

Figure S2 ${ }^{1} \mathrm{H}$ NMR spectrum of $\underline{\mathbf{2 b}}$ 